九州大学学術情報リポジトリ

Kyushu University Institutional Repository

\title{
日本の地域一般住民における一過性脳虚血発作の発 症率、危険因子、予後の時代的推移：久山町研究
}

\section{古田，芳彦}

ht tp://hdl. hand le. net/2324/2198516

出版情報: Kyushu University，2018，博士（医学），課程博士 バージョン：

権利関係 : 


\title{
Secular trends in the incidence, risk factors, and prognosis of transient ischemic attack in Japan: The Hisayama Study
}

\author{
Yoshihiko Furuta a, b, Jun Hata a, b, c, *, Naoko Mukai a, b, c, Yoichiro Hirakawa a, b,

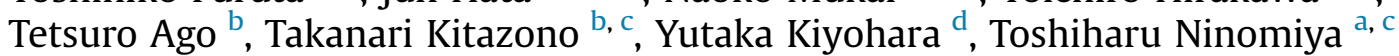 \\ a Department of Epidemiology and Public Health, Graduate School of Medical Sciences, Kyushu University, 3-1-1 Maidashi, Higashi-ku, Fukuoka 812-8582, \\ Japan \\ ${ }^{\mathrm{b}}$ Department of Medicine and Clinical Sciences, Graduate School of Medical Sciences, Kyushu University, 3-1-1 Maidashi, Higashi-ku, Fukuoka 812-8582, \\ Japan \\ ${ }^{\mathrm{C}}$ Center for Cohort Studies, Graduate School of Medical Sciences, Kyushu University, 3-1-1 Maidashi, Higashi-ku, Fukuoka 812-8582, Japan \\ ${ }^{\mathrm{d}}$ Hisayama Research Institute for Lifestyle Diseases, 1822-1 Kubara, Hisayama-machi, Kasuya-gun, Fukuoka 811-2501, Japan
}

\section{A R T I C L E I N F O}

\section{Article history:}

Received 24 January 2018

Received in revised form

28 March 2018

Accepted 11 April 2018

Available online 12 April 2018

\section{Keywords:}

Transient ischemic attack

Incidence

Epidemiology

Cohort study

Risk factors

\begin{abstract}
A B S T R A C T
Background and aims: We aimed to investigate secular trends in the incidence, risk factors, and prognosis of transient ischemic attack (TIA) in a general Japanese population.

Methods: Two cohorts consisting of stroke-free Japanese individuals aged $\geq 40$ years in $1961(\mathrm{n}=1621)$ and 1988 ( $n=2646)$ were followed up for 24 years. The associations of potential risk factors with the development of TIA were estimated by a Cox proportional hazards model. The influence of TIA on the risk of total stroke over the subsequent 10 years was compared between the sub-cohorts of subjects with TIA and age- and sex-matched control subjects from each cohort.

Results: During follow-up, 28 subjects in the 1961 cohort and 34 in the 1988 cohort experienced TIA. The age-standardized incidence of TIA was significantly lower in the 1988 cohort than the 1961 cohort (0.66 vs. 1.01 per 1000 person-years, $\mathrm{p}=0.02$ ). While elevated systolic blood pressure was significantly associated with the risk of TIA in both cohorts, glucose intolerance and higher serum cholesterol levels were associated with TIA risk only in the 1988 cohort. The subjects experiencing TIA had approximately 7 -8 -fold higher risks for the 10-year incidence of total and ischemic strokes compared with the corresponding control subjects without TIA both in the 1961 and 1988 sub-cohorts, and the relative risks were not significantly different between sub-cohorts.

Conclusions: Our results suggest that the incidence of TIA decreased during the past half century, probably due to the spread of antihypertensive treatments in the general Japanese population.
\end{abstract}

(C) 2018 Elsevier B.V. All rights reserved.

\section{Introduction}

Transient ischemic attack (TIA) is a clinically important symptom as an early warning sign of imminent stroke. Several population-based observational studies [1-14] have estimated the incidence of TIA, but most of them [2-9,11-14] were performed in Western countries. In Asia, there has only been our previous report in conjunction with the Hisayama Study [15], in which we examined the incidence of TIA in the period from 1961 to 1981 in a

\footnotetext{
* Corresponding author. Department of Epidemiology and Public Health, Graduate School of Medical Sciences, Kyushu University, 3-1-1 Maidashi, Higashi-ku, Fukuoka 812-8582, Japan.

E-mail address: junhata@eph.med.kyushu-u.ac.jp (J. Hata).
}

general Japanese population. Over the last half century, the prevalence and severity of cardiovascular risk factors in Japan, such as hypertension, diabetes, dyslipidemia, and smoking, have dramatically changed [16], and the management of these risk factors has been improved. Consequently, the incidence rate of stroke has been decreased in Japan [16]. Therefore, it can be assumed that the incidence of TIA in the Japanese population has also changed with time. However, this issue has not been adequately addressed in Western countries [1-3], and has been almost completely ignored in Japan. Moreover, no studies have examined the secular trends in the association between risk factors and the development of TIA and in the risk of subsequent stroke after TIA. The aim of the present study was to estimate the secular trends in the incidence, risk factors, and prognosis of TIA in a Japanese community, Hisayama, 
using two cohorts separated by an interval of 27 years.

\section{Materials and methods}

\subsection{Study population}

The Hisayama Study is a population-based cohort study of cardiovascular diseases (CVD) established in 1961. The town of Hisayama, where the epidemiological study has been ongoing, is located in a suburb of the Fukuoka metropolitan area in Kyushu Island, Japan. According to the national census, the population of the town was approximately 6500 in 1960 and 8400 in 2010. The age and occupational distributions and nutrient intake levels in the residents of Hisayama have been similar to those of Japan as a whole during the past half century [16]. A detailed description of this study was published previously [15-18]. A total of 1658 residents of Hisayama aged $\geq 40$ years participated in the health examination in 1961 (participation rate in this age group: 90.1\%) $[16,17]$. We excluded 4 participants who died and 8 who moved away from the town before the start of follow-up, and 25 who had a history of stroke, and selected the remaining 1621 participants as the 1961 cohort. Similarly, a total of 2742 Hisayama residents aged $\geq 40$ years underwent the health examination in 1988 (participation rate: 80.9\%) [18]. After excluding 6 participants who died before the start of follow-up and 90 who had a history of stroke, the remaining 2646 participants were included in the 1988 cohort. This study was approved by the Kyushu University Institutional Review Board for Clinical Research, and written or oral informed consent was obtained from all the participants.

\subsection{Risk factors}

Blood pressure was measured three times in a supine position in 1961 and in a sitting position in 1988 using a mercury sphygmomanometer after an at least 5-min rest. Korotkoff phase 5 was taken as the diastolic blood pressure unless the sounds persisted at zero, in which case Korotkoff phase 4 was recorded. The mean of the three measurements was used in the present study. Hypertension was defined as blood pressure $\geq 140 / 90 \mathrm{mmHg}$ or use of antihypertensive agents. In 1961, glucose intolerance was defined by a full stomach test or $100-\mathrm{g}$ oral glucose tolerance test (OGTT) in participants with glycosuria as described previously [16]. In 1988, we performed 75-g OGTT for almost all participants aged 40-79 years, or a fasting or postprandial glucose measurement for others [18]. In this cohort, glucose intolerance, which included both diabetes and prediabetes, was defined as fasting plasma glucose concentration $\geq 6.1 \mathrm{mmol} / \mathrm{L}, \quad 2-\mathrm{h}$ postload plasma glucose concentration $\geq 7.8 \mathrm{mmol} / \mathrm{L}$, postprandial plasma glucose concentration $\geq 11.1 \mathrm{mmol} / \mathrm{L}$, or use of antidiabetic agents. Serum total cholesterol levels were measured by the modified Zak-Henly method in 1961 and by the enzymatic method in 1988 [16]. Hypercholesterolemia was defined as serum total cholesterol $\geq 5.7 \mathrm{mmol} / \mathrm{L}$. Overweight was defined as body mass index $\geq 25 \mathrm{~kg} / \mathrm{m}^{2}$. Atrial fibrillation was diagnosed by electrocardiogram (Minnesota code: $8-3$ ). Smoking habits and alcohol intake were categorized as either current use or not. Current drinking was also categorized as light (1-33 g/day) or excessive ( $\geq 34 \mathrm{~g} /$ day) drinking according to daily ethanol intake.

\subsection{Follow-up survey}

The participants of each cohort were followed up prospectively for 24 years, from November 1961 to October 1985 for the 1961 cohort, and from December 1988 to November 2012 for the 1988 cohort, by the same method. In the annual follow-up examinations, physicians and research nurses asked each participant whether he/ she had experienced clinical symptoms of CVD events (TIA, stroke, and coronary heart disease). For participants who did not undergo a regular examination or who moved from the town, vital status and clinical information of CVD were collected by mail or telephone interviews. In addition, in order to collect the information of potential CVD events more exhaustively, we established a daily monitoring system among the study team, local physicians, and members of the town's Health and Welfare Office. When a participant died, autopsy examination was performed at Kyushu University if consent for autopsy was obtained. All of the available information about potential CVD events or deaths among the study participants were collected and reviewed by physician members of the Hisayama Study, including stroke neurologists and cardiologists, to determine the occurrence of CVD under the standardized diagnostic criteria. All CVD events were adjudicated by means of a panel discussion among member physicians. Except for the deceased cases, only two participants in the 1961 cohort and no participants in the 1988 cohort were lost to follow-up during the follow-up periods.

\subsection{Definition of TIA}

TIA was defined as a sudden onset of nonconvulsive focal neurological deficit, such as hemiparesis, aphasia, amaurosis fugax, and so on, due to brain ischemia which resolved within $24 \mathrm{~h}$, regardless of the presence or absence of corresponding ischemic stroke lesions on brain imaging or autopsy, according to the Classification of Cerebrovascular Disease III (CVD-III) criteria by the National Institute of Neurological Disorders and Stroke published in 1990 [19]. During the follow-up periods, 28 participants in the 1961 cohort and 34 in the 1988 cohort experienced a TIA event.

\subsection{Cumulative risk of stroke after TIA}

In order to compare cumulative risks of total stroke and ischemic stroke after the onset of TIA between the two cohorts, we established a sub-cohort consisting of subjects who developed TIA during the 24-year follow-up and control subjects who were randomly selected from the participants without TIA events and matched by baseline age and sex in each cohort (case:control =1:3). The 1961 sub-cohort included 28 subjects with TIA and 84 control subjects, and the 1988 sub-cohort had 28 TIA subjects (excluding 6 subjects who developed TIA after a stroke event during the follow-up) and 84 controls. Each sub-cohort was followed up for 10 years from the date of TIA onset for each subject with TIA (or the corresponding date for matched control subjects) or until October 1985 or November 2012. Stroke was defined as a sudden onset of focal neurological deficit persisting for $>24$ years and classified as ischemic stroke and hemorrhagic stroke (intracerebral and subarachnoid hemorrhage) according to the CVD-III criteria [19]. Ischemic stroke events were further divided into lacunar infarction, atherothrombotic infarction, cardioembolic infarction, and other ischemic stroke according to the Trial of Org 10172 in Acute Stroke Treatment criteria [20], as described previously [21].

\subsection{Statistical analysis}

The prevalence of risk factors was adjusted for age and sex by the direct method using the distribution in the 1961 cohort as a reference population, and compared between the two cohorts by logistic regression analysis. The mean values of risk factors were adjusted for age and sex and compared between the cohorts by the analysis of covariance. The incidence of TIA was calculated by the person-years method, and the standardization for age was made by 
the direct method using the WHO Standard Population [22]. To investigate the association between cardiovascular risk factors and the risk of TIA, the age- and sex-adjusted hazard ratio (HR) and its 95\% CI were estimated by a Cox proportional hazards model. For each sub-cohort, cumulative risks of total and ischemic strokes were estimated and compared by the marginal proportional hazards model proposed by Lin and Wei [23]. Heterogeneity in the HRs for the development of stroke between the 1961 and the 1988 subcohorts was evaluated by adding an interaction term into the Cox model. The SAS 9.4 (SAS Institute Inc., Cary, North Carolina, USA) was used to perform all statistical analyses. Two-sided values of $p<0.05$ were considered statistically significant.

\section{Results}

Table 1 shows the baseline characteristics of participants in the 1961 and 1988 cohorts after adjustment for age and sex. Mean age was significantly higher in the 1988 cohort than in the 1961 cohort. The prevalence of hypertension was constant, but the mean values of systolic blood pressure in total subjects and the mean values of systolic and diastolic blood pressures in subjects with hypertension decreased significantly from the 1961 cohort to the 1988 cohort, as a result of the spread of antihypertensive treatment. While the proportion of current smokers and current drinkers decreased significantly with time, the prevalence of glucose intolerance, hypercholesterolemia, overweight, and excessive drinking increased significantly.

Table 2 presents the incidence of TIA in the two cohorts. In the 1961 cohort, the age-standardized incidence of TIA per 1000 person-years was 1.01 in total, 1.55 for men, and 0.64 for women; and that in the 1988 cohort was $0.66,1.09$, and 0.42 , respectively. The age-standardized incidence declined significantly from the 1961 cohort to the 1988 cohort in total $(p=0.02)$ and for men $(p=0.04)$. A similar declining trend was observed for women but was not statistically significant $(p=0.21$ ), probably due to the small number of events ( $p=0.75$ for heterogeneity between men and women). In both cohorts, the age-standardized incidence of TIA was significantly higher in men than in women $(p<0.01$ for both cohorts), and the age-specific incidence of TIA was increased significantly with aging ( $p<0.05$ for both cohorts).

Table 3 demonstrates the age- and sex-adjusted HRs of risk factors for the development of TIA in each cohort. Higher systolic blood pressure was significantly associated with the risk of TIA in both cohorts, while glucose intolerance and higher serum cholesterol levels were significant risk factors only in the 1988 cohort.

We investigated the influence of TIA on the risk of total stroke and ischemic stroke over the subsequent 10 years among the subcohorts of subjects with TIA and age- and sex-matched control subjects. As shown in Fig. 1, the subjects with TIA had approximately 7-8-fold higher risks of total and ischemic stroke compared with the corresponding control subjects both in the 1961 and 1988 sub-cohorts, and the magnitude of the influence of TIA on the subsequent risks of total and ischemic stroke was not significantly different between the sub-cohorts (both $p$ for heterogeneity $>0.8$ ). In addition, the age- and sex-adjusted 10-year incidence of total and ischemic stroke after TIA seemed to be lower in the 1988 subcohort than in the 1961 sub-cohort, but the difference did not reach the level of statistical significance (both $p>0.3$ ).

Among the 28 subjects with TIA in the 1961 sub-cohort, 13 experienced ischemic stroke, and 2 experienced hemorrhagic stroke during 10 years of follow-up. Among the 28 subjects with TIA in the 1988 sub-cohort, 12 had ischemic stroke, and 2 had hemorrhagic stroke. Fig. 2 shows the proportion of ischemic stroke subtypes in subjects with TIA from each sub-cohort. The proportion of ischemic stroke subtypes was $31 \%(n=4)$ for atherothrombotic infarction, 38\% $(n=5)$ for lacunar infarction, and 31\% $(n=4)$ for cardioembolic infarction in the 1961 sub-cohort, and the corresponding values in the 1988 sub-cohort were $58 \%(n=7), 25 \%$ $(\mathrm{n}=3)$, and $17 \%(\mathrm{n}=2)$.

\section{Discussion}

In the present study, the age-standardized incidence of TIA clearly decreased during the past half century. To the best of our knowledge, this is the first observational study that examined secular trends in the incidence of TIA in a Japanese community. With regard to risk factors for TIA, higher systolic blood pressure was a consistent risk factor for TIA both in the 1961 and the 1988 cohorts, while higher serum cholesterol levels and glucose intolerance were associated with the risk of TIA only in the 1988 cohort. Subjects with TIA had significantly higher risks of total and ischemic strokes than those without TIA in both cohorts, and the

Table 1

Age- and sex-adjusted baseline characteristics of the study participants in the 1961 and 1988 cohorts.

\begin{tabular}{|c|c|c|c|}
\hline & $\begin{array}{l}\text { The } 1961 \text { cohort } \\
(n=1621)\end{array}$ & $\begin{array}{l}\text { The } 1988 \text { cohort } \\
(n=2646)\end{array}$ & $p$ value \\
\hline Age, years (sex-adjusted) & $56(0.29)$ & $59(0.23)$ & $<0.001$ \\
\hline Men, \% (age-adjusted) & 43.6 & 42.6 & 0.60 \\
\hline Hypertension, \% & 38.0 & 38.7 & 0.85 \\
\hline Antihypertensive agents, \% & 2.2 & 13.2 & $<0.001$ \\
\hline $\mathrm{SBP}, \mathrm{mmHg}$ & $137(0.54)$ & $133(0.42)$ & $<0.001$ \\
\hline $\mathrm{DBP}, \mathrm{mmHg}$ & $78(0.30)$ & $78(0.24)$ & 0.45 \\
\hline SBP in hypertensive individuals, $\mathrm{mmHg}$ & $162(0.74)$ & $153(0.55)$ & $<0.001$ \\
\hline DBP in hypertensive individuals, $\mathrm{mmHg}$ & $89(0.43)$ & $85(0.32)$ & $<0.001$ \\
\hline Glucose intolerance, \% & 8.0 & 34.2 & $<0.001$ \\
\hline Hypercholesterolemia, \% & 5.1 & 35.3 & $<0.001$ \\
\hline Total cholesterol, mmol/L & $4.05(0.03)$ & $5.32(0.02)$ & 0.04 \\
\hline Overweight, \% & 10.6 & 24.3 & $<0.001$ \\
\hline Body mass index, $\mathrm{kg} / \mathrm{m}^{2}$ & $21.5(0.07)$ & $22.9(0.06)$ & $<0.001$ \\
\hline Atrial fibrillation, \% & 0.7 & 1.1 & 0.16 \\
\hline Current smoker, \% & 42.7 & 26.1 & $<0.001$ \\
\hline Current drinker, \% & 34.9 & 32.7 & 0.045 \\
\hline Excessive drinker, \% & 11.4 & 15.7 & $<0.001$ \\
\hline
\end{tabular}

SBP, systolic blood pressure; DBP, diastolic blood pressure.

Values are mean (standard error of the mean) or a percentage. 
Table 2

Incidence of TIA (per 1000 person-years) during 24-year follow-up of the 1961 and 1988 cohorts.

\begin{tabular}{|c|c|c|c|c|c|}
\hline & \multicolumn{2}{|l|}{$\begin{array}{l}\text { The } 1961 \text { cohort } \\
(1961-1985)\end{array}$} & \multicolumn{2}{|l|}{$\begin{array}{l}\text { The } 1988 \text { cohort } \\
(1988-2012)\end{array}$} & \multirow{2}{*}{$\begin{array}{l}p \text { value } \\
\text { (1961 vs. 1988) }\end{array}$} \\
\hline & Events/subjects & Incidence & Events/subjects & Incidence & \\
\hline \multicolumn{6}{|l|}{ Total } \\
\hline Crude incidence & $28 / 1621$ & 0.96 & $34 / 2646$ & 0.67 & 0.14 \\
\hline Age-standardized incidence & & 1.01 & & 0.66 & 0.02 \\
\hline \multicolumn{6}{|l|}{ Age-specific incidence } \\
\hline 40-49 years & $7 / 551$ & 0.57 & $2 / 685$ & 0.13 & \\
\hline $50-59$ years & $13 / 479$ & 1.35 & $8 / 745$ & 0.50 & \\
\hline $60-69$ years & $5 / 362$ & 0.94 & $12 / 668$ & 0.94 & \\
\hline$\geq 70$ years & $3 / 229$ & 1.53 & $12 / 548$ & 1.87 & \\
\hline \multicolumn{6}{|l|}{ Men } \\
\hline Crude incidence & $18 / 707$ & 1.50 & $20 / 1115$ & 0.99 & 0.19 \\
\hline Age-standardized incidence & & 1.55 & & 1.09 & 0.04 \\
\hline \multicolumn{6}{|l|}{ Age-specific incidence } \\
\hline $40-49$ years & $5 / 246$ & 0.95 & $2 / 304$ & 0.29 & \\
\hline $50-59$ years & $8 / 216$ & 1.94 & $3 / 318$ & 0.46 & \\
\hline $60-69$ years & $4 / 168$ & 1.91 & $8 / 285$ & 1.60 & \\
\hline$\geq 70$ years & $1 / 77$ & 1.80 & $7 / 208$ & 3.58 & \\
\hline \multicolumn{6}{|l|}{ Women } \\
\hline Crude incidence & $10 / 914$ & 0.58 & $14 / 1531$ & 0.46 & 0.51 \\
\hline Age-standardized incidence & & 0.64 & & 0.42 & 0.21 \\
\hline \multicolumn{6}{|l|}{ Age-specific incidence } \\
\hline 40-49 years & $2 / 305$ & 0.29 & $0 / 381$ & 0 & \\
\hline $50-59$ years & $5 / 263$ & 0.90 & $5 / 427$ & 0.52 & \\
\hline $60-69$ years & $1 / 194$ & 0.31 & $4 / 383$ & 0.51 & \\
\hline$\geq 70$ years & $2 / 152$ & 1.43 & $5 / 340$ & 1.12 & \\
\hline
\end{tabular}

Table 3

Age- and sex-adjusted HRs of risk factors for the development of TIA in the 1961 and 1988 cohorts.

\begin{tabular}{|c|c|c|c|c|c|c|}
\hline & \multicolumn{3}{|c|}{$\begin{array}{l}\text { The } 1961 \text { cohort } \\
(1961-1985)\end{array}$} & \multicolumn{3}{|c|}{$\begin{array}{l}\text { The } 1988 \text { cohort } \\
(1988-2012)\end{array}$} \\
\hline & HR & $(95 \% \mathrm{CI})$ & $p$ & HR & $(95 \% \mathrm{CI})$ & $p$ \\
\hline Age (per 10-year increase) & 1.68 & $(1.12-2.51)$ & 0.01 & 2.30 & $(1.66-3.19)$ & $<0.001$ \\
\hline Men ( $v s$. women) & 2.96 & $(1.36-6.46)$ & 0.01 & 2.68 & $(1.34-5.36)$ & 0.005 \\
\hline SBP (per $10 \mathrm{mmHg}$ increase) & 1.16 & $(1.00-1.35)$ & 0.046 & 1.19 & $(1.03-1.38)$ & 0.02 \\
\hline Glucose intolerance (yes vs. no) & 0.38 & $(0.05-2.84)$ & 0.35 & 2.12 & $(1.07-4.17)$ & 0.03 \\
\hline Total cholesterol (per $1 \mathrm{mmol} / \mathrm{L}$ increase) & 0.98 & $(0.65-1.48)$ & 0.93 & 1.49 & $(1.12-1.98)$ & 0.006 \\
\hline Body mass index (per $1 \mathrm{~kg} / \mathrm{m}^{2}$ increase) & 0.97 & $(0.97-1.13)$ & 0.68 & 1.07 & $(0.96-1.20)$ & 0.22 \\
\hline Current smoker (yes vs. no) & 0.42 & $(0.18-1.01)$ & 0.05 & 1.81 & $(0.82-3.99)$ & 0.14 \\
\hline Current drinker (yes vs. no) & 0.79 & $(0.33-1.94)$ & 0.61 & 1.16 & $(0.51-2.64)$ & 0.72 \\
\hline Excessive drinker (yes vs. no) & 1.21 & $(0.43-3.41)$ & 0.72 & 0.78 & $(0.29-2.15)$ & 0.63 \\
\hline
\end{tabular}

HR, hazard ratio; CI, confidence interval; SBP, systolic blood pressure.

\section{A Total stroke}

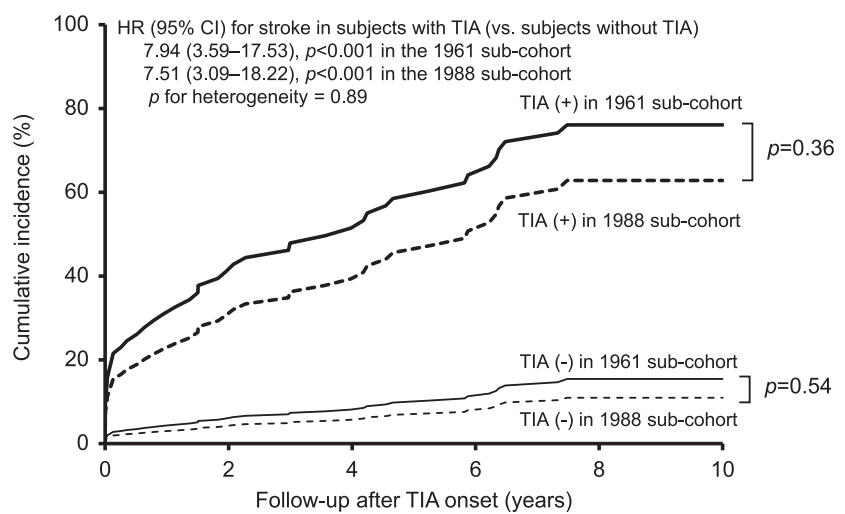

B Ischemic stroke

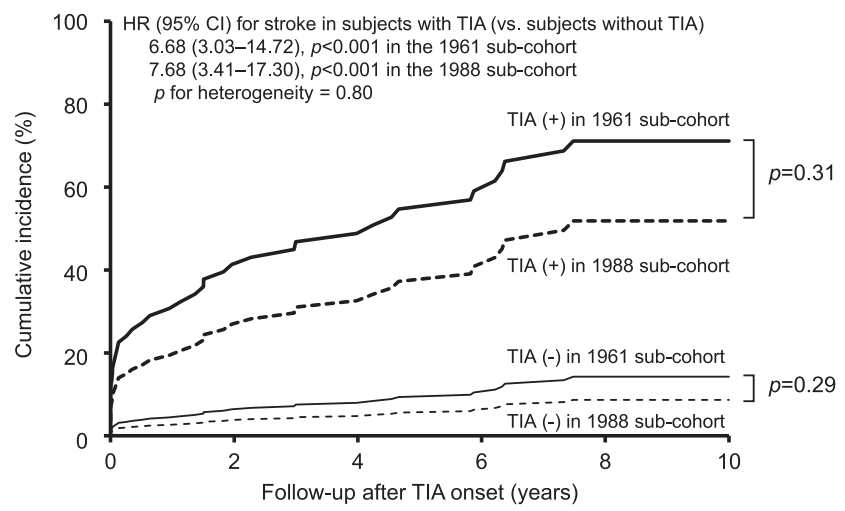

Fig. 1. Secular change in the age- and sex-adjusted cumulative incidence of stroke after TIA.

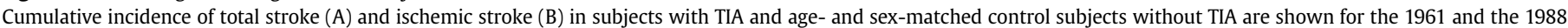

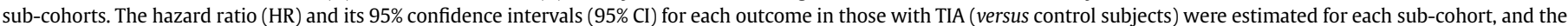
heterogeneity in the magnitude of the association between TIA and the risk of each outcome was calculated. 

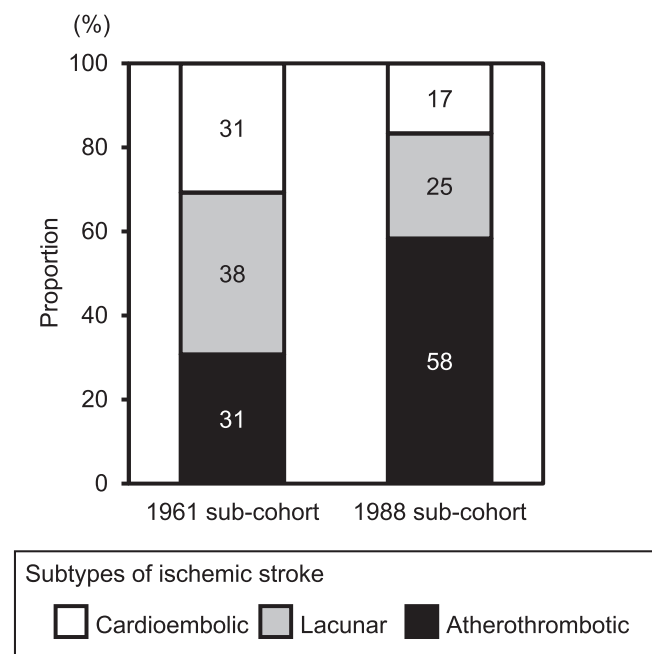

Fig. 2. Secular change in the proportion of ischemic stroke subtypes developed after TIA.

The proportion of ischemic stroke subtypes developed over a period of 10 years is shown in subjects with TIA for the 1961 and 1988 sub-cohorts.

magnitude of this association did not change between the cohorts. Our results would provide useful information on the primary prevention of TIA and stroke after TIA in the general Japanese population.

Several registry-based studies from other countries have reported the annual incidence of TIA among total populations including all age groups: the annual incidence of TIA varied from 0.05 to 1.4 per 1000 persons [1-10]. Some prospective cohort studies of Western adult populations have also examined the incidence of TIA [12-14]. The Rotterdam Study from the Netherlands showed that the TIA incidence in individuals aged $\geq 55$ years was 4.7 per 1000 person-years [12]. In the cohort study for individuals aged $\geq 50$ years in Rochester, Minnesota, the TIA incidence was 2.37 per 1000 population [13]. The incidence of our Japanese individuals aged $\geq 40$ years ( 1.01 per 1000 person-years in the 1961 cohort and 0.66 in the 1988 cohort) seemed to be lower than those in the Western studies. In the 1960s, a similar lower incidence was observed in a cohort study of American men with Japanese ancestry aged 45-68 years (average annual incidence, 1.08 per 1000 population) [11]. These findings suggest that the incidence of TIA was lower in Japanese than in the Western populations, although a direct comparison among the studies is difficult because of differences in the age distribution and study methods, including the methods of follow up. These differences, in turn, may have been due to the fact that extracranial atherosclerosis is less common in Japanese than Westerners [24].

A few registry-based studies have shown the secular trends in the incidence of TIA. In Novosibirsk, Russia, the incidence of TIA in all age groups did not increase significantly (0.16 per 1000 population in 1987-1988 and 0.29 per 1000 in 1996-1997) [1]. A similar stable trend in the incidence of TIA was observed in a registration study in France (0.12 per 1000 in $1985-1989$ and 0.11 per 1000 in 2000-2004) [2]. Meanwhile, the present cohort study revealed a clear trend of decrease in the TIA incidence from the 1961 cohort to the 1988 cohort of middle-aged and elderly Japanese individuals. In our population, the mean values of systolic and diastolic blood pressures in hypertensive subjects decreased significantly during this period as a result of the spread of antihypertensive treatment. Therefore, the decreasing trends in the TIA incidence between the two cohorts could be mainly explained by the improvement of hypertension management.

In our population, subjects who experienced TIA events had a higher subsequent incidence of total and ischemic stroke than those without TIA both in the 1961 and the 1988 sub-cohorts. Intriguingly, the present study revealed that the proportion of lacunar infarction among subsequent ischemic stroke after TIA decreased with time, while the proportion of atherothrombotic infarction increased. Lacunar infarction usually occurs by the occlusion of small perforating arteries mainly in hypertensive subjects. In contrast, atherothrombotic infarction is caused by atherosclerotic disease of large arteries, such as the carotid and intracranial arteries. In the 1988 cohort, metabolic risk factors, namely, glucose intolerance and elevated serum cholesterol levels, were significant risk factors for TIA, but they were not associated with the risk of TIA in the 1961 cohort. These changes of risk factors for TIA are attributable to the increasing trends in the prevalence of metabolic disorders with time, due to the westernization of dietary habits and physical inactivity as a result of motorization [16]. Since diabetes [25] and hypercholesterolemia [26] are known risk factors for atherosclerotic disease, our finding suggests that the etiology of TIA may have shifted from small artery diseases to large artery diseases. Notably, the magnitude of the relative risk of TIA for subsequent stroke did not decrease significantly with time in the present study, despite the improvement in the management of hypertension, possibly because the increased burden of metabolic risk factors offset the benefit of improved management of hypertension.

The present study had some notable advantages. First, we performed a population-based survey of TIA events which were confirmed by a combination of several methods, including face-toface interview by physicians and research nurses at the annual health examination, questionnaire by mail or telephone, and information from the local physicians and town government, because TIA patients do not always visit hospitals. Therefore, we consider that our population-based cohort study could provide more accurate estimations of the TIA incidence than hospital-based registry studies. Second, participation rates of the baseline examinations in 1961 and in 1988 were high, and thus the selection bias in these cohorts was minimal.

However, several limitations should also be noted. First, we could not estimate the association between atrial fibrillation and the risk of TIA, because no subjects with atrial fibrillation at baseline experienced TIA during the follow-up period in either cohort, and we could not investigate the association between other risk factors and the risk of TIA using multivariable or stratified analyses, due to the small number of TIA event. In addition, since the number of ischemic stroke events after TIA was very small, it was difficult to investigate the association between TIA and subsequent ischemic stroke in more detail. Second, we could not determine the etiology of TIA, because available information on the underlying vascular diseases or embolic sources was insufficient due to the populationbased study setting. Third, the definition of glucose intolerance and the methods used to measure serum cholesterol levels were different between the two cohorts. The diagnosis of glucose intolerance and the measurement of serum cholesterol in the 1961 cohort might have been less accurate than those in the 1988 cohort. These limitations might have led to underestimation of the influence of glucose intolerance and high serum cholesterol levels on the incidence of TIA, especially in the 1961 cohort. Finally, detailed information of risk factors, treatment (e.g., antithrombotic and anticoagulant therapy), and comorbidity (e.g., carotid artery lesions) at the time of TIA onset was not available. Therefore, we could not evaluate the influence of risk factors on subsequent stroke events after TIA. 


\subsection{Conclusions}

In a cohort study of a general Japanese population, the incidence of TIA was shown to have decreased over the past half century, probably due to the improvement in hypertension treatment. However, the influence of metabolic disorders, such as glucose intolerance and hypercholesterolemia, on the risk of TIA increased during this period. As a consequence, the proportion of atherothrombotic stroke increased among stroke cases developed after TIA. Since the prevalence of metabolic risk factors has been increasing progressively with time due to westernized lifestyles and physical inactivity as a result of motorization in Japanese [16], we cannot deny the possibility that the TIA incidence will plateau or trend upward rather than downward in the near future. Intensive efforts for the comprehensive management of metabolic risk factors as well as hypertension will be needed to reduce the burden of TIA and subsequent stroke in the future.

\section{Conflicts of interest}

The authors declared they do not have anything to disclose regarding conflict of interest with respect to this manuscript.

\section{Financial support}

This study was supported in part by Grants-in-Aid for Scientific Research (A) (JP16H02644, and JP16H02692) and (B) (JP16H05850, JP16H05557, and JP17H04126) and (C) (JP15K09267, JP15K08738, JP15K09835, JP16K09244, JP17K09114, JP17K09113, and JP17K01853) from the Ministry of Education, Culture, Sports, Science and Technology of Japan; by Health and Labour Sciences Research Grants of the Ministry of Health, Labour and Welfare of Japan (H25-Junkankitou [Seishuu]-Sitei-022, H29-JunkankitouIppan-003, and H27-Shokuhin-[Sitei]-017); and by the Japan Agency for Medical Research and Development (dk0207025, ek0210082, gm0610007, ek0210083, km0405202, ek0210080).

\section{Author contributions}

Yoshihiko Furuta: study concept and design, analysis and interpretation of data, acquisition of data, writing of the manuscript.

Jun Hata: study concept and design, interpretation of data, acquisition of data, critical revision of the manuscript for intellectual content.

Naoko Mukai: interpretation of data, acquisition of data, critical revision of the manuscript for intellectual content.

Yoichiro Hirakawa: interpretation of data, acquisition of data, critical revision of the manuscript for intellectual content.

Tetsuro Ago: interpretation of data, critical revision of the manuscript for intellectual content.

Takanari Kitazono: interpretation of data, critical revision of the manuscript for intellectual content, study supervision.

Yutaka Kiyohara: study concept and design, interpretation of data, acquisition of data, critical revision of the manuscript for intellectual content, study supervision.

Toshiharu Ninomiya: study concept and design, interpretation of data, acquisition of data, critical revision of the manuscript for intellectual content, study supervision.

\section{Acknowledgements}

We thank the staff of the Division of Health and Welfare of Hisayama for their cooperation in this study. We also would like to gratefully and sincerely thank Professor Yoshinao Oda, Professor
Toru Iwaki, and the staff of the Department of Anatomic Pathology and Department of Neuropathology, Graduate School of Medical Sciences, Kyushu University, who provided insight and expertise in regard to the autopsy findings that greatly assisted the research.

\section{References}

[1] V.L. Feigin, S.V. Shishkin, G.M. Tzirkin, T.E. Vinogradova, A.V. Tarasov, et al., A population-based study of transient ischemic attack incidence in Novosibirsk, Russia, 1987-1988 and 1996-1997, Stroke 31 (2000) 9-13, https:// doi.org/10.1161/01.str.31.1.9.

[2] Y. Bejot, O. Rouaud, I. Benatru, J. Durier, M. Caillier, et al., Trends in the incidence of transient ischemic attacks, premorbid risk factors and the use of preventive treatments in the population of Dijon. France from 1985 to 2004, Cerebrovasc. Dis. 23 (2007) 126-131, https://doi.org/10.1159/000097049.

[3] G.D. Friedman, W.S. Wilson, J.M. Mosier, M.A. Colandrea, M.Z. Nichaman, Transient ischemic attacks in a community, J. Am. Med. Assoc. 210 (1969) $1428-1434$.

[4] M.S. Dennis, J.M. Bamford, P.A. Sandercock, C.P. Warlow, Incidence of transient ischemic attacks in Oxfordshire, England, Stroke 20 (1989) 333-339.

[5] R.D. Brown, G.W. Petty, W.M. O'Fallon, D.O. Wiebers, J.P. Whisnant, Incidence of transient ischemic attack in Rochester, Minnesota, 1985-1989, Stroke 29 (1998) 2109-2113, https://doi.org/10.1161/01.str.29.10.2109.

[6] D. Kleindorfer, P. Panagos, A. Pancioli, J. Khoury, B. Kissela, et al., Incidence and short-term prognosis of transient ischemic attack in a population-based study, Stroke $36 \quad$ (2005) 720-723, https://doi.org/10.1161/ 01.STR.0000158917.59233.b7.

[7] M. Correia, M.R. Silva, R. Magalhaes, L. Guimaraes, M.C. Silva, Transient ischemic attacks in rural and urban northern Portugal: incidence and shortterm prognosis, Stroke 37 (2006) 50-55, https://doi.org/10.1161/ 01.STR.0000195209.26543.8f

[8] M.F. Giles, P.M. Rothwell, Substantial underestimation of the need for outpatient services for TIA and minor stroke, Age Ageing 36 (2007) 676-680, https://doi.org/10.1093/ageing/afm088.

[9] I. Cancelli, F. Janes, G.L. Gigli, A. Perelli, B. Zanchettin, et al., Incidence of transient ischemic attack and early stroke risk: validation of the ABCD2 score in an Italian population-based study, Stroke 42 (2011) 2751-2757, https:// doi.org/10.1161/STROKEAHA.110.612705.

[10] P.G. Fonseca, P.A. Weiss, R. Harger, C.H. Moro, A.L. Longo, et al., Transient ischemic attack incidence in Joinville, Brazil, 2010: a population-based study, Stroke $43 \quad$ (2012) 1159-1162, https://doi.org/10.1161| STROKEAHA.111.645101.

[11] G.G. Rhoads, J.S. Popper, A. Kagan, K. Yano, Incidence of transient cerebral ischemic attack in Hawaii Japanese men. the honolulu heart study, Stroke 11 (1980) 21-26.

[12] M.J. Bos, M.J. van Rijn, J.C. Witteman, A. Hofman, P.J. Koudstaal, et al., Incidence and prognosis of transient neurological attacks, J. Am. Med. Assoc. 298 (2007) 2877-2885, https://doi.org/10.1001/jama.298.24.2877.

[13] J.P. Whisnant, L.J. Melton 3rd, P.H. Davis, W.M. O'Fallon, K. Nishimaru, et al. Comparison of case ascertainment by medical record linkage and cohort follow-up to determine incidence rates for transient ischemic attacks and stroke, J. Clin. Epidemiol. 43 (1990) 791-797.

[14] C. Weikert, K. Berger, C. Heidemann, M.M. Bergmann, K. Hoffmann, et al., Joint effects of risk factors for stroke and transient ischemic attack in a German population: the EPIC potsdam study, J. Neurol. 254 (2007) 315-321, https:// doi.org/10.1007/s00415-006-0358-X.

[15] K. Ueda, Y. Kiyohara, Y. Hasuo, T. Yanai, H. Kawano, et al., Transient cerebral ischemic attacks in a Japanese community, Hisayama, Japan, Stroke 18 (1987) 844-848, https://doi.org/10.1161/01.str.18.5.844.

[16] J. Hata, T. Ninomiya, Y. Hirakawa, M. Nagata, N. Mukai, et al., Secular trends in cardiovascular disease and its risk factors in Japanese: half-century data from the Hisayama Study, Circulation 2013 (128) (1961-2009) 1198-1205, https:// doi.org/10.1161/CIRCULATIONAHA.113.002424.

[17] S. Katsuki, Epidemiological and clinicopathological study on cerebrovascular disease in Japan, Prog. Brain Res. 21 (1966) 64-89.

[18] M. Fujishima, Y. Kiyohara, I. Kato, T. Ohmura, H. Iwamoto, et al., Diabetes and cardiovascular disease in a prospective population survey in Japan: the Hisayama Study, Diabetes 45 (Suppl 3) (1996) S14-S16.

[19] Special report from the national institute of neurological disorders and stroke. classification of cerebrovascular diseases III, Stroke 21 (1990) 637-676.

[20] H.P. Adams Jr., B.H. Bendixen, L.J. Kappelle, J. Biller, B.B. Love, et al., Classification of subtype of acute ischemic stroke: definitions for use in a multicenter clinical trial. TOAST. trial of org 10172 in acute stroke treatment, Stroke 24 (1993) 35-41.

[21] Y. Tanizaki, Y. Kiyohara, I. Kato, H. Iwamoto, K. Nakayama, et al., Incidence and risk factors for subtypes of cerebral infarction in a general population: the Hisayama Study, Stroke 31 (2000) 2616-2622.

[22] O.B. Ahmad, C. Boschi-Pinto, A.D. Lopez, C.J.L. Murray, R. Lozano, et al., Age Standardization of Rates: a New WHO Standard, GPE Discussion Paper Series, WHO, Geneva, 2001.

[23] L.J. Wei, D.Y. Lin, L. Weissfeld, Regression analysis of multivariate incomplete failure time data by modeling marginal distributions, J. Am. Stat. Assoc. 84 (1989) 1065-1073, https://doi.org/10.2307/2290084. 
[24] S.A. Kieffer, Y. Takeya, J.A. Resch, K. Amplatz, Racial differences in cerebrovascular disease: angiographic evaluation of Japanese and American populations, Am. J. Roentgenol. Radium Ther. Nucl. Med. 101 (1967) 94-99.

[25] A.I. Qureshi, L.R. Caplan, Intracranial atherosclerosis, Lancet 383 (2014) 984-998, https://doi.org/10.1016/S0140-6736(13)61088-0.
[26] T. Imamura, Y. Doi, H. Arima, K. Yonemoto, J. Hata, et al., LDL cholesterol and the development of stroke subtypes and coronary heart disease in a general Japanese population: the Hisayama study, Stroke 40 (2009) 382-388, https:// doi.org/10.1161/STROKEAHA.108.529537. 